\title{
Development of regulatory loop for the quantitative control of gene expression
}

\author{
Vasilev R.A. ${ }^{1,2 *}$, Polushkina I.D. ${ }^{1,3}$ \\ ${ }^{1}$ National Research Center «Kurchatov Institute», Moscow, Russia \\ ${ }^{2}$ Lomonosov Moscow State University, Moscow, Russia \\ ${ }^{3}$ D.Mendeleev University of Chemical Technology of Russia, Moscow, Russia \\ *e-mail: ruavasilev@gmail.com
}

Key words: artificial circuits, RNA-switch, regulatory loop, intein splicing, RNA interference

Motivation and Aim: Gene therapy methods development has a great potential in medicine applications due to the ability to influence the etiological factors of diseases caused by mutations. In the same time there are an amount of nosologies etiology of those is based on quantitative changes in expression of particular genes. In this order development of integrated to genome targeted quantitative gene expression regulation tools is a worthy of note way to the therapy of the particular diseases. Hereditary hypercholesterolemia which is caused by apolipoprotein B (apoB) transcripts amount's bias is one of such diseases instances[1]. To modulate apoB expression we are planning to assemble a closed feedback system with posttranscriptional regulation of apoB.

Methods and Algorithms: We are developing 2 model systems. The first one can be represented as a feedback loop with an apoB-100 expression level posttranscriptionally regulated by interference with miRNA. In turn miRNA transcription can be initiated in the presence of chimeric transcription factor StdCas9-VP64. Translation of this TF will happen only in the case of additional activation: 5'-end secondary structure destabilization of mRNA. This can be achieved by introduction of toehold-like RNAswitch directly upstream of IRES in 5'-UTR of StdCas9-VP64. Expressed apoB-100 transcripts play translation activators role «unwrapping» the RNA-switch. In the second model system we offer to utilize intein splicing in trans instead of the RNA-switch. Using CRISPR/Cas it is possible to fuse 5'UTR of apoB100 with N-terminal domain of chimeric TF flanked by N-intein. C-terminal domain of StdCas9-VP64 with $\mathrm{C}$-intein will be expressed constitutively. As a result we are going to obtain closed multicomponent system with a feedback loop. To test the obtained RNA-switch for translation regulation capability we assembled genetic construction which encode RNA-switch and downstream-located EGFP gene and tried it in the coupled in vitro transcription |translation system [2].

Results: Genetic constructions with RNA-switch and inteins were assembled. RNA-switch was shown to implement translation regulation in the expected manner: in the absence of trigger mRNA ( $5^{\prime}$-UTR of ApoB mRNA) EGFP production was inhibited in the coupled in vitro transcription।translation system, on the other hand EGFP protein level was observed to be analogous to positive control (EGFP native mRNA).

Conclusion: Current results suggest that developed RNA-switch can be utilized as translation regulation tool and can be further used in mammalian cell-culture studies.

Acknowledgements: Supported by the RFBR (No. 18-29-07046).

\section{References}

1. Vega G. L. et al. In vivo evidence for reduced binding of low density lipoproteins to receptors as a cause of primary moderate hypercholesterolemia. Journal Clinical Investigation. 1986. V. 78. №. 5. P. 1410-1414.

2. Pelham H. R. B., Jackson R. J. An efficient mRNA-dependent translation system from reticulocyte lysates. European Journal Biochemistry. 1976. V. 67. №. 1. P. 247-256. 